\title{
Cardiovascular Events in Early Rheumatoid Arthritis - Role of Tumor Necrosis Factor Inhibition
}

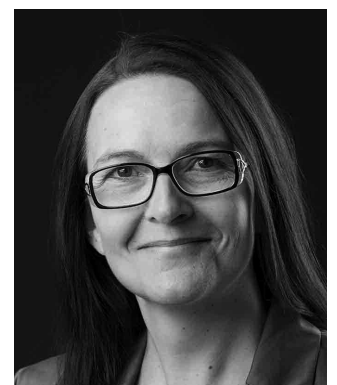

Cardiovascular (CV) comorbidity is frequent among patients with rheumatoid arthritis (RA) ${ }^{1}$. This is most marked regarding coronary disease, with an often-observed doubled risk of acute myocardial infarction in patients with RA compared with the general population ${ }^{1}$. Although the pathogenic mechanisms behind the increased risk of $\mathrm{CV}$ events remain to be explained, associations with traditional $\mathrm{CV}$ risk factors, as well as factors related to RA disease activity and severity, have been shown ${ }^{2}$. With disease-related risk factors being of importance, the effect of traditional risk factors on $\mathrm{CV}$ risk is lower in RA versus the general population ${ }^{3}$. Further, in the light of active RA disease, interpretation of risk factors can be complex, as illustrated by the effect of inflammation on blood lipids, or the effects of smoking on development of both RA and CV disease $2,4,5$. The high degree of systemic inflammation in active RA disease is associated with premature and accelerated atherosclerosis, a process that underlies many $\mathrm{CV}$ events ${ }^{6}$.

Several studies evaluating newly diagnosed patients with RA have shown a rapid increase in CV risk after disease debut $^{7}$. Accumulated disease activity during the earliest stages of RA disease has been associated with risk of CV events, and reduction in disease activity during the first year with an improved prognosis ${ }^{8,9}$. It has been suggested that there is a "window of opportunity" soon after disease debut, where control of inflammation can modify the risk of $\mathrm{CV}$ comorbidity ${ }^{10}$. The importance of inflammatory control is further emphasized by the association of treatment with synthetic disease-modifying antirheumatic drugs (DMARD) with a reduced risk of $\mathrm{CV}$ disease in both established and early RA, as observed in several studies ${ }^{8,9,11}$. Because biological DMARD such as tumor necrosis factor inhibitors (TNFi) are not only efficient in lowering disease activity but also might block cytokines immediately involved in the atherosclerotic process, these therapies have gained extra interest. Several studies in established RA or in RA of varying or undefined duration have observed TNFi exposure to be associated with a decreased risk of $\mathrm{CV}$ events ${ }^{12,13}$; however, data from patients with early RA are scarce. In a Swedish national cohort study comprising 6000 patients with early RA, we observed TNF inhibitor treatment not to be statistically significantly associated with the risk of acute coronary syndrome (HR $0.8,95 \%$ CI $0.5-1.2)^{14}$. Because to date this was the only observational study of the risk of $\mathrm{CV}$ disease and TNFi in early RA, the nested case-control study by Desai and colleagues in this issue of The Journal is a welcome contribution to the scientific field ${ }^{15}$.

The objective of their study was to examine, in an early $\mathrm{RA}$ population, risk of $\mathrm{CV}$ events associated with $\mathrm{TNFi}$ therapy ${ }^{15}$. The base cohort of patients starting a TNFi or a nonbiological DMARD for early RA 2008-2010 was identified from an insurance database. From this cohort, 279 cases with early RA and an incident CV event (defined as a diagnosis of acute or stable ischemic heart disease, chronic heart failure, or cerebrovascular event) were assembled, with 12 matched controls each. The risk of CV events among patients with ongoing TNFi treatment, previous TNFi treatment, or previous nonbiological DMARD therapy, respectively, was compared with the risk among patients with ongoing nonbiological DMARD. The analyses were adjusted for covariates including traditional $\mathrm{CV}$ risk factors (e.g. diabetes mellitus, hypertension, and hyperlipidemia), other comorbidity, and pharmacotherapy. In this study the risk of $\mathrm{CV}$ events did not differ among patients with early RA and ongoing TNF inhibitor treatment compared with ongoing synthetic DMARD therapy. In patients without previous TNFi exposure, past use of nonbiologic DMARD was associated with a higher risk of $\mathrm{CV}$ events.

Possible explanations for the different results observed in studies of associations between $\mathrm{CV}$ risk and TNFi exposure are discussed by the present authors, noting a number of methodological differences hampering comparisons, besides the obvious difference in disease duration. There are, however, other reasons why a possible difference

See TNF- $\alpha$ inhibitors and risk of incident CVD in early RA, page 2129

Personal non-commercial use only. The Journal of Rheumatology Copyright $(\subset) 2014$. All rights reserved. 
in the CV risk could remain unnoted in this study and also in others' when evaluating early RA populations.

Even though the evidence is convincing that there is an increased risk of $\mathrm{CV}$ events also in early RA, it can take some time for this risk to be detectable. There are seemingly differences between types of $\mathrm{CV}$ events regarding when in the disease process the increase in risk evolves. In a Swedish cohort study, an over-risk of acute myocardial infarction was detectable in an early RA population after 1 year, and in a second study, of stroke after about a decade ${ }^{16,17}$. Because the data in the present study do not include specific clinical findings, exact disease duration is not known, but the mean followup of less than 1 year might be somewhat short regarding the compound outcome.

Further, using active treatment as a prerequisite for inclusion might lead to a risk of selection of early RA patients with better general health and a lower risk of CV events for the base cohort. Clinically, if a patient is considered to be at an immediate high risk of serious comorbidity, this is likely to affect treatment decisions. Because all patients in this study were receiving active treatment, the incidence of $\mathrm{CV}$ events in this specific base cohort might be lower than in other studied cohorts, taking into account the possible "window of opportunity."

As recommended, and as seen in clinical practice, in most patients with early RA the rheumatologist will start treatment with a synthetic DMARD or DMARD combination and complement it with, or change the medication to, a biological DMARD if the response is insufficient ${ }^{18}$. The present study lacks data on disease activity or response to treatment, but if we assume that the patients have been treated to achieve low disease activity or remission (whether or not biologics have been used) and that the CV risk is related to inflammatory activity, the contrast between the actively treated groups might not be detectable.

There might also be differences between treatment groups regarding the baseline risk. It is unknown whether the inflammatory activity at inclusion in the base cohort was comparable between groups or if the TNFi exposed group(s) had higher disease activity, and thus a possibly higher background $\mathrm{CV}$ risk at baseline. Tendency of a lower point estimate in sensitivity analysis, adjusted for proxy measures of disease severity, could indicate this. We also do not know whether the presumably impaired primary therapy response among the patients who started TNFi therapy as a second-line option, and the possibly longer time period of active disease in this group, had any effect on CV risk.

The case-control design of this study resulted in a higher mean age (64 years) among the included patients than is usually found in an early RA inception cohort, which is natural because age is an important risk factor for incident $\mathrm{CV}$ events. Age, however, seems to have an effect on treatment decisions, as well as disease activity measurements. Late-onset RA (> 58 yrs) was observed to be associated with higher disease activity, more disability, and a higher frequency of erosions in the early phase ${ }^{19}$. Regarding treatment, corticosteroids were used more frequently and synthetic and biologic DMARD less often among patients with late onset. Another recent study described how disease-related factors varied as predictors of $\mathrm{CV}$ events in younger and older patients ${ }^{9}$. The differences in treatment could be a signal that comorbidities or general frailty more frequently affecting the older patient could influence treatment decisions, resulting in confounding by (contra-)indication that might be difficult to fully adjust for.

Other limitations of this well-performed study have been discussed by the authors, and even though they have taken some of these remarks into account through sensitivity analyses, they conclude in the discussion that the existing evidence is not sufficient. Even with a meticulous study design, observational studies have limitations, and there is still a need for larger, prospective, observational studies of this area.

With the knowledge we have today, with the exception of the contraindications in congestive heart failure, we could consider TNFi safe to use from a CV perspective, because no study, including this one, has reported a statistically significantly increased CV risk with TNFi exposure. We can also be quite certain that if any mitigating effect of TNFi on the $\mathrm{CV}$ risk in early RA exists, it will be of modest strength, and possibly not clinically relevant in the early RA population as a whole. The higher risk of $\mathrm{CV}$ events observed in the group of early RA patients who discontinued DMARD supports the theory of the importance of early and maintained inflammatory control; which for the clinician, in fact, may be the most important lesson from this work.

LOTTA LJUNG, MD, PhD,

Department of Public Health and Clinical Medicine/Rheumatology, Umeå University, Umeå; and

Department of Medicine, Clinical Epidemiology Unit,

Karolinska Institutet,

Stockholm, Sweden.

Address correspondence to Dr. Ljung, Department of Rheumatology, Umeå University Hospital, S-901 85 Umeå, Sweden.

E-mail: lotta.ljung@vll.se

\section{REFERENCES}

1. Avina-Zubieta JA, Thomas J, Sadatsafavi M, Lehman AJ, Lacaille D. Risk of incident cardiovascular events in patients with rheumatoid arthritis: a meta-analysis of observational studies. Ann Rheum Dis 2012;71:1524-9.

2. Choy E, Ganeshalingam K, Semb AG, Szekanecz Z, Nurmohamed M. Cardiovascular risk in rheumatoid arthritis: recent advances in the understanding of the pivotal role of inflammation, risk predictors and the impact of treatment. Rheumatology 2014 June 6 (E-pub ahead of print).

3. Gonzalez A, Maradit Kremers H, Crowson CS, Ballman KV, Roger VL, Jacobsen SJ, et al. Do cardiovascular risk factors confer the same risk for cardiovascular outcomes in rheumatoid arthritis patients as in non-rheumatoid arthritis patients? Ann Rheum Dis

Personal non-commercial use only. The Journal of Rheumatology Copyright @ 2014 . All rights reserved. 
2008;67:64-9.

4. Costenbader KH, Feskanich D, Mandl LA, Karlson EW. Smoking intensity, duration, and cessation, and the risk of rheumatoid arthritis in women. Am J Med 2006;119:503 e1-9.

5. Yusuf S, Hawken S, Ounpuu S, Dans T, Avezum A, Lanas F, et al. Effect of potentially modifiable risk factors associated with myocardial infarction in 52 countries (the INTERHEART study): case-control study. Lancet 2004;364:937-52.

6. del Rincon I, Williams K, Stern MP, Freeman GL, O'Leary DH, Escalante A. Association between carotid atherosclerosis and markers of inflammation in rheumatoid arthritis patients and healthy subjects. Arthritis Rheum 2003;48:1833-40.

7. Kerola AM, Kauppi MJ, Kerola T, Nieminen TV. How early in the course of rheumatoid arthritis does the excess cardiovascular risk appear? Ann Rheum Dis 2012;71:1606-15.

8. Innala L, Möller B, Ljung L, Magnusson S, Smedby T, Södergren A, et al. Cardiovascular events in early RA are a result of inflammatory burden and traditional risk factors: a five year prospective study. Arthritis Res Ther 2011;13:R131.

9. Ajeganova S, Andersson ML, Frostegard J, Hafström I. Disease factors in early rheumatoid arthritis are associated with differential risks for cardiovascular events and mortality depending on age at onset: a 10-year observational cohort study. J Rheumatol 2013;40:1958-66.

10. Liang KP. Cardiovascular risk in rheumatoid arthritis (RA): does it matter if RA is diagnosed in early or late age [editorial]? J Rheumatol 2013;40:1945-7.

11. van Halm VP, Nurmohamed MT, Twisk JW, Dijkmans BA, Voskuyl AE. Disease-modifying antirheumatic drugs are associated with a reduced risk for cardiovascular disease in patients with rheumatoid arthritis: a case control study. Arthritis Res Ther 2006;8:R151.

12. Barnabe C, Martin BJ, Ghali WA. Systematic review and meta-analysis: Anti-tumor necrosis factor alpha therapy and cardiovascular events in rheumatoid arthritis. Arthritis Care Res 2011;63:522-9.
13. Westlake SL, Colebatch AN, Baird J, Curzen N, Kiely P, Quinn M, et al. Tumour necrosis factor antagonists and the risk of cardiovascular disease in patients with rheumatoid arthritis: a systematic literature review. Rheumatology 2011;50:518-31.

14. Ljung L, Simard JF, Jacobsson L, Rantapää-Dahlqvist S, Askling J. Treatment with tumour necrosis factor-inhibitors and the risk of acute coronary syndromes in early rheumatoid arthritis. Arthritis Rheum 2012;64:42-52.

15. Desai R, Rao J, Hansen R, Fang G, Maciejewski M, Farley J. TNF-alpha inhibitor treatment and the risk of incident cardiovascular events in patients with early rheumatoid arthritis: a nested case-control study. J Rheumatol 2014;41:2129-36.

16. Holmqvist ME, Wedren S, Jacobsson LT, Klareskog L, Nyberg F, Rantapää-Dahlqvist $\mathrm{S}$, et al. Rapid increase in myocardial infarction risk following diagnosis of rheumatoid arthritis amongst patients diagnosed between 1995 and 2006. J Intern Med 2010;268:578-85.

17. Holmqvist M, Gränsmark E, Mantel A, Alfredsson L, Jacobsson LT, Wållberg-Jonsson S, et al. Occurrence and relative risk of stroke in incident and prevalent contemporary rheumatoid arthritis. Ann Rheum Dis 2013;72:541-6.

18. Singh JA, Furst DE, Bharat A, Curtis JR, Kavanaugh AF, Kremer JM, et al. 2012 update of the 2008 American College of Rheumatology recommendations for the use of disease-modifying antirheumatic drugs and biologic agents in the treatment of rheumatoid arthritis [review]. Arthritis Care Res 2012;64:625-39.

19. Innala L, Berglin E, Möller B, Ljung L, Smedby T, Södergren A, et al. Age at onset determines severity and choice of treatment in early rheumatoid arthritis: a prospective study. Arthritis Res Ther 2014;16:R94.

J Rheumatol 2014;41:2094-6; doi:10.3899/jrheum.140838 